\title{
Métodos de elaboración de guías de onda
}

\author{
Plamen Neytchev Nechev \\ Jaime Castellanos Corredor \\ Profesores Departamento de Física \\ Universidad Pedagógica Nacional
}

En este artículo de divulgación se considera la construcción de dos tipos de guías de onda planas, en cuya superficie se puede propagar la luz en cualquier dirección y de bandas o "canales", elaborados en forma especial sobre su superficie y por los cuales únicamente puede propagarse la luz.

\section{GUIAS DE ONDA PLANAS:}

Los métodos para su elaboración se pueden agrupar en dos:

1. Se deposita una película sobre el sustrato de manera que el perfil del índice de refracción resulte escalonado.

2. En la capa justo por debajo de la superficie de separación aire-sustrato se hace variar el índice de refracción así que el perfil óptico es del tipo de gradiente.

Una técnica usada desde 1973 consiste en el intercambio iónico en vidrio que tiene la ventaja de disminuir las pérdidas de propagación de la luz y facilitar la conexión con fibras ópticas, debido a la semejanza de los materiales y de los índices de refracción.

El intercambio iónico de algunos vidrios con ciertas sustancias se origina en la presencia de iones móviles de $\mathrm{Na}$ que se difunden fácilmente en el vidrio y pueden intercambiar sus posiciones con otros iones de la solución del fundido, que se encuentra en contacto directo con el sustrato de vidrio. Vidrios de este tipo contienen por lo general $\mathrm{Na}_{2} \mathrm{O}, \mathrm{K}_{2} \mathrm{O}$ ó $\mathrm{Li}_{2} \mathrm{O}$. Teniendo en cuenta otros elementos, cuya presencia es indispensable en los vidrios, se concluye que las mejores posibilidades de intercambio las poseen iones de valencia uno como $\mathrm{Ag}^{+}, \mathrm{Li}^{+}, \mathrm{Na}^{+}, \mathrm{K}^{+}, \mathrm{Te}^{+}$. Una composición típica (en pesos) es: $\mathrm{SiO}_{2}(71,56 \%)$, óxidos alcalinos, $\mathrm{Na}_{2} \mathrm{O}, \mathrm{K}{ }_{2} \mathrm{O}(13,33 \%), \quad \mathrm{CaO}(8,69 \%), \quad \mathrm{MgO}(4,03 \%)$, $\mathrm{Al}_{2} \mathrm{O}_{3}(1,25 \%), \mathrm{Fe}_{2} \mathrm{O}_{3}(\mathrm{O}, 14 \%)$.

La difusión de los iones puede describirse en forma simplificada por la ecuación

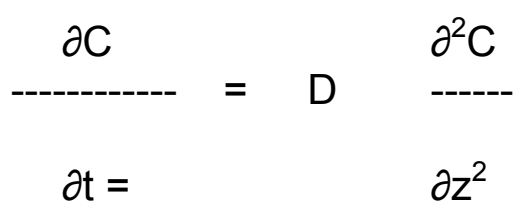

Esta ecuación se aplica principalmente para el intercambio de iones de plata que junto con el intercambio de los de sodio son los procesos mejor comprendidos.

No obstante la ecuación describe el mecanismo ftsico solamente. Aquí C es la concentración de los iones que se difunden, $D$ es el coeficiente de auto-difusión de los iones de plata y $z$ es la profundidad de penetración en el sustrato. 
En la fabricación de la guía primero se realiza un proceso de intercambio de iones sumergiendo la placa de vidrio en un recipiente de cuarzo que contiene, entre $220^{\circ} \mathrm{C}$ y $260^{\circ} \mathrm{C}$, nitrato de plata $\left(\mathrm{AgNO}_{3}\right)$ fundido de manera que se forma una guía de onda plana en cada cara del sustrato.

En la figura 1 se muestra el perfil del Índice de refracción $n(z)$ para una guía de onda de 7 modos. También se muestra para comparación la curva teórica de Gauss y la curva parabólica. El perfil real se obtiene con ayuda del espectro modal neff. 7 que también se muestra en la figura.

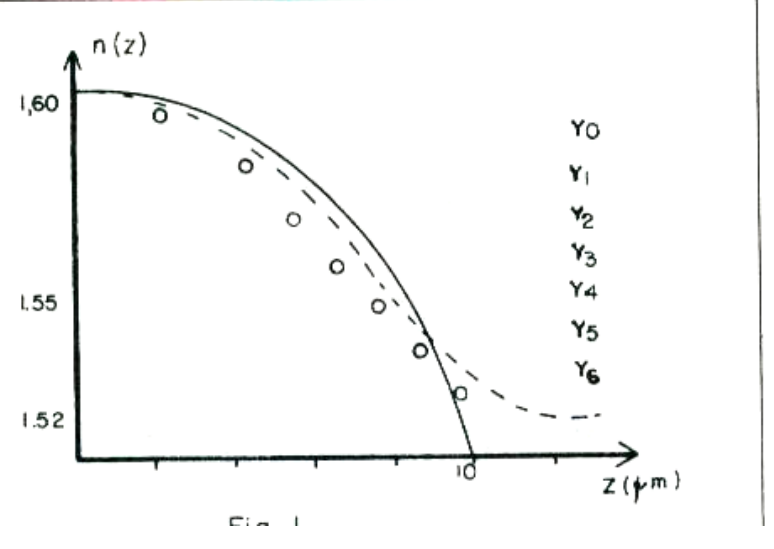

Fig. I

El intercambio jónico en el vidrio se caracteriza por un incremento alto del índice de refracción del sustrato $(\mathrm{Dn}=10 \%)$, por tener su valor máximo sobre la superficie y porque la velocidad de creación de los modos es relativamente pequeña aún para temperaturas altas, aproximadamente 1 ó 2 modos por hora.

Para mejorar el proceso anterior se utiliza un fundido en el cual el nitrato de plata está muy disuelto en nitrato de sodio $\left(\mathrm{NaNO}_{3}\right)$. En este caso la solución de la ecuación de difusión lleva a la siguiente expresión para la concentración de los iones $\mathrm{Ag}^{+}$en vidrio.

$$
\mathrm{C}_{(\mathrm{Z}, \mathrm{T})=\mathrm{co}} \operatorname{erf}(\mathrm{Z} / \mathrm{D})
$$

donde Co es la concentración de los iones de plata en el fundido y $\mathrm{D}$ se determina por $\mathrm{D}=(\mathrm{tD} .)^{1 / 2}$

La dependencia entre Dn y Co hallada experimentalmente para vidrio con un 10 a $15 \%$ de $\mathrm{Na}_{2} \mathrm{O}$ se muestra en la figura 2, donde puede verse una dependencia para valores pequeños de Co. 


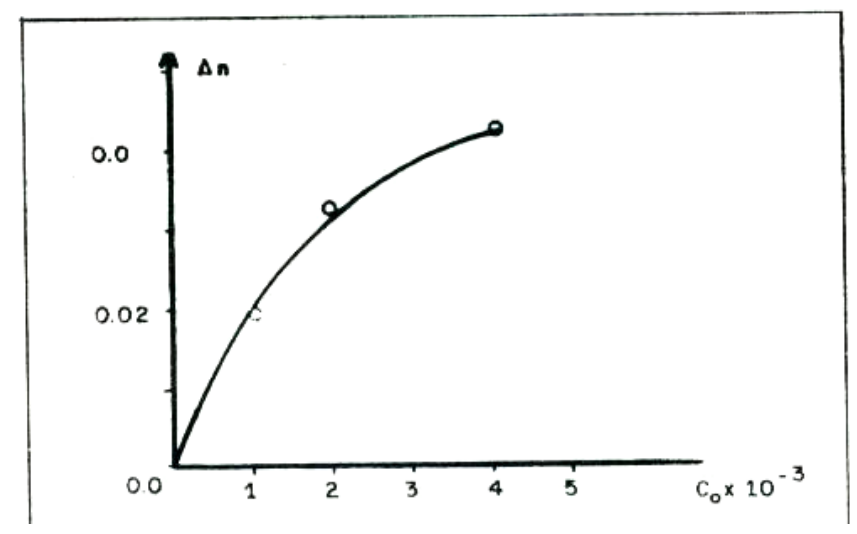

Fig. 2

La dependencia del coeficiente de difusión $\mathrm{D}$ con la temperatura tiene la forma $\mathrm{D}_{\mathrm{T}}=\mathrm{Do} \mathrm{e}^{-\mathrm{AH} / \mathrm{RT}}$

donde $\mathrm{DH}$ es la energía de activación y $\mathrm{R}$ es la constante universal de los gases.

Tomando logaritmos

$$
\mathrm{LnD}_{\mathrm{T}}=-\Delta \mathrm{H} / \mathrm{RT}+\operatorname{InDo}
$$

de tal manera que $\Delta H / R$ es la pendiente de esta recta. De la figura 3 se puede obtener

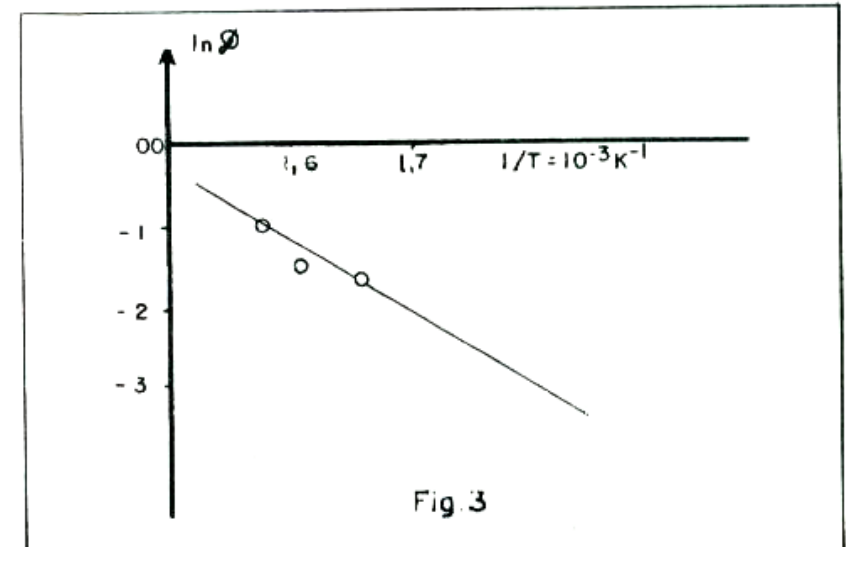

Fig. 3

$$
\begin{aligned}
& \Delta H=8,9333 \times 10^{4} \mathrm{~J} \\
& D o=1,1906 \times 10^{-7 m 2 / s}
\end{aligned}
$$


Para la misma concentración de iones $\mathrm{Ag}^{+}$y diferente tiempo de difusión se obtienen películas de diferentes espesores, pero con el mismo Dn y por consiguiente guías de onda con diferente número de modos.

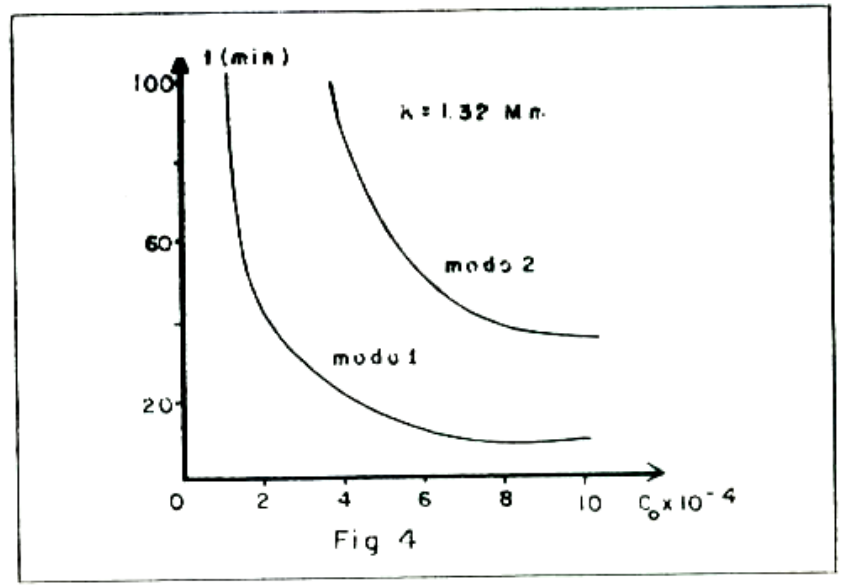

Fig. 4

La relación entre Co y t para los diferentes modos, con base en la ec.(1) se puede ver en las figuras 4 y 5 .

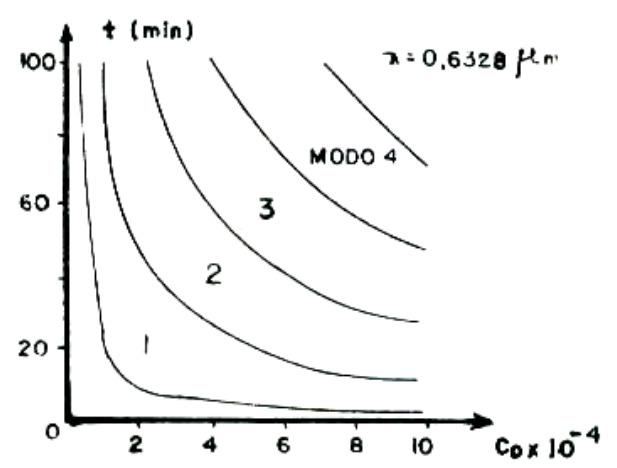

Fig. 5

Fig. 5

Ambas gráficas presentan la misma relación para longitudes de onda de $1,32 \mathrm{~mm}$ y $0,6328 \mathrm{~mm}$. Con su ayuda se pueden diseñar guías de onda de modos fijados con anticipación. Por ejemplo, al fijar el tiempo de difusión (que se determina por el espesor necesario de la guía), de estas figuras, se puede encontrar el valor de Co que asegura la guía de onda monomodo.

La posibilidad de obtener guías de onda con niobiato de litio $\left(\mathrm{LiNbQ}_{3}\right)$ se basa en el método de difusión inversa. Al calentar al vacío durante varias horas el sustrato a $1100^{\circ}$ $\mathrm{C}$, de la superficie sale $\mathrm{LiO}_{2}$ que deja una capa de espesor de 50 a $150 \mathrm{~mm}$. A pesar de 
tener un buen espesor, su aplicación es limitada, debido a la aparición del haz extraordinario.

Para guías electroópticas se utiliza difusión térmica de $\mathrm{Ti}$ en $\mathrm{LiNbO}_{3}$. El titanio se coloca sobre la superficie del sustrato de niobiato de litio en una capa muy delgada (10 a $50 \mathrm{~nm}$ ) por pulverización magnética y luego durante varias horas se realiza la difusió $\mathrm{n}$ a $1000^{\circ} \mathrm{C}$ aproximadamente. La penetración del titanio en el sustrato lleva a un incremento de 0,01 del índice de refracción tanto del ordinario $n_{0}$, como del extraordinario $n_{e}$ (fig.6).

Sobre las causas de aparición de guías ópticas de $\mathrm{Ti}_{\mathrm{LiNbO}}$ no existe opinión única. Las investigaciones estructurales muestran que los iones de titanio ocupan los puestos de Li o de $\mathrm{Nb}$, lo cual lleva a una deformación de la red cristalina del niobiato de litio debido a los diferentes radios. Probablemente esta deformación induce un cambio en los índices de refracción $n_{o}$ y $n_{e}$ debido a efectos fotoelásticos.

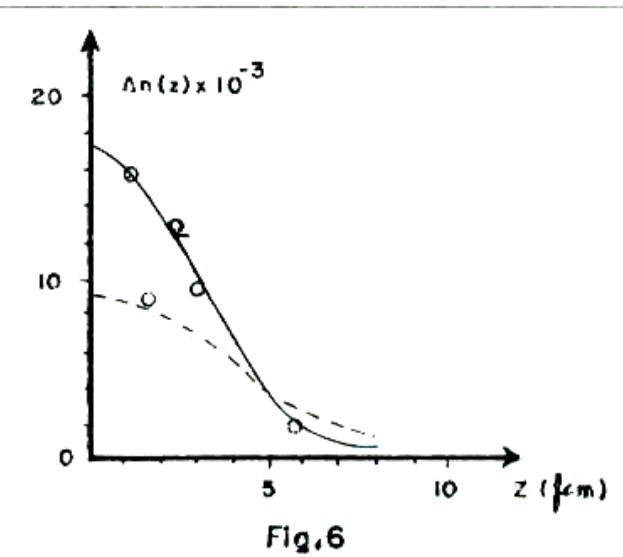

Fig. 6

Cada modo tiene su atenuación propia y la intensidad de la luz decae exponencialmente en la dirección de propagación

$$
I=I_{0} \exp \left(-x X_{m}\right)
$$

En el caso general de atenuación total $x_{t}$ para un cierto modo $m$, tiene dos componentes, la dispersión superficial $X_{s s} y$ la atenuación volumétrica $X_{v}$, que a su turno se causa por dispersión $\mathrm{X}_{\mathrm{vs}} \mathrm{y}$ absorción $\mathrm{X}_{\mathrm{a}}$ dentro del volumen de la capa.

$$
X_{t}=X_{s s}+X_{v}=X_{s s}+X_{v}=X_{s s}+X_{v s}+X_{a}
$$

Por lo general las pérdidas se miden con ayuda de una fibra óptica que se desplaza a lo largo de la huella luminosa. Al determinar atenuaciones débiles, del orden de $0,1 \mathrm{~dB} / \mathrm{cm}$, es necesario utilizar instrumentos sensibles, como contadores de fotones, controlando las variaciones de la intensidad de la luz incidente ya que no pueden evitarse. 


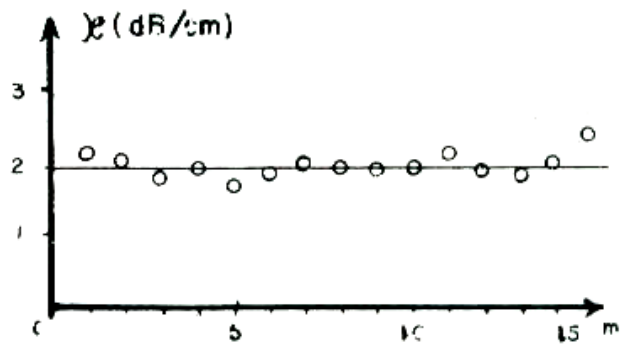

Fiq 7

A diferencia de las guías homogéneas, la atenuación total en guías de gradiente casi no depende del orden del modo. Algunos resultados típicos para guías de onda obtenidas por intercambio iónico en vidrio se muestran en la fig.7. Un análisis muestra que la función $\mathrm{X}_{\mathrm{t}(\mathrm{m})}$ es la suma del componente $\mathrm{X}_{\mathrm{ss}(\mathrm{m})}$ que aumenta linealmente, y el componente $\mathrm{X}_{\mathrm{v}(\mathrm{m})}$ que disminuye linealmente también, por lo tanto es posible una compensación parcial o total.

\section{GUIAS DE ONDA DE BANDA:}

Las guías de banda son los principales elementos de la óptica integrada que permiten construir circuitos ópticos integrados y complejos. El proceso de construcción comienza con la formación de una fotomáscara apropiada. Con ayuda de un patrón generador se dibuja la estructura requerida a través de un haz luminoso sobre una fotoplaca sensible en escala 1:1. La longitud de los dispositivos ópticos integrados y por consiguiente la longitud de estos circuitos es mucho mayor que su ancho.

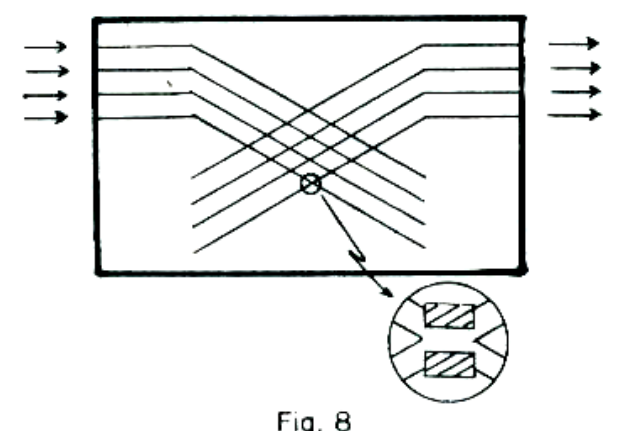

Fig. 8

Fig. 8

Por ejemplo, una matriz de conmutación apreciablemente compleja (fig.8) que contiene 16 conmutadores ópticos ocupa un área de aproximadamente $50 \mathrm{~mm}$ de longitud y solo $0,5 \mathrm{~mm}$ de ancho. Por esta desproporción de las dimensiones la elaboración de la máscara crea dificultades considerables. Desde el punto de vista de la fotomáscara resultan más cómodas las estructuras de forma cuadrada, así que esto obliga a utilizar guías con algunas partes curvas.

Para disminuir las pérdidas de luz el radio de la curvatura debe ser mayor de $5 \mathrm{~mm}$. 
La estructura que contiene la fotomáscara se transplanta sobre el sustrato con ayuda de una máquina de exposición especial de alta resolución (fig. 9). Sobre el sustrato 5 se coloca con anticipación una capa sensible a la luz que se llama fotoresistor. El sustrato está en una cámara de vacío y se tapa con la fotomáscara, cuyo contacto directo se logra al hacer el vacío. Con un haz ultravioleta paralelo se pueden reproducir estructuras con exactitud mayor de $] \mu_{m}$ Luego viene la serie de procedimientos fotográficos ordinarios que llevan poco a poco a la formación de las bandas de la guía.

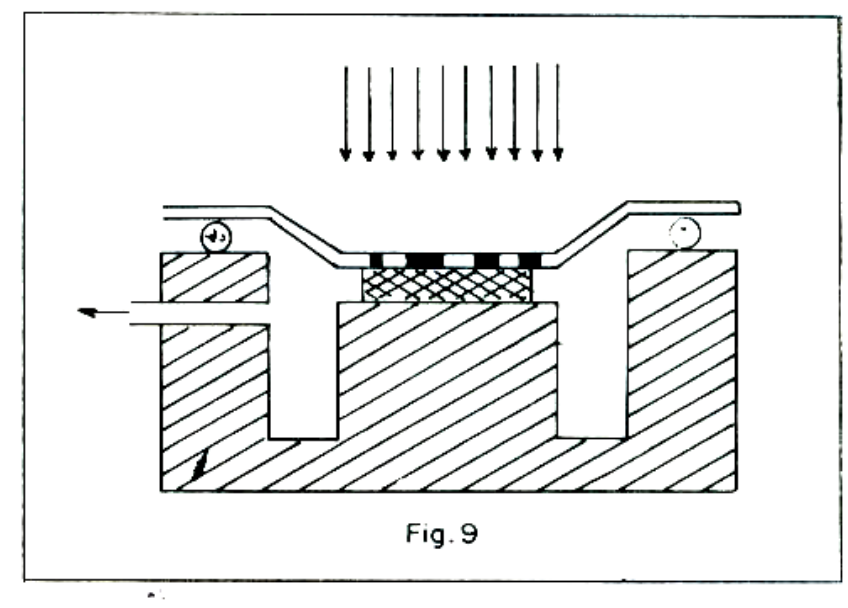

Fig. 9

Como ilustración en la fig. 10 se muestran las sucesivas etapas de la construcción de guías de banda de $\mathrm{Ti}_{\mathrm{LiNbO}}$. 'En la figura se ha supuesto un fotoresistor positivo, lo que significa que las regiones bajo exposición se lavan con ayuda de un revelador. Si se trabaja con un fotoresistor negativo se lavan las regiones no expuestas. En la fig. 10 a se esquematiza el llamado proceso litográfico "lift-off". El mismo resultado final se puede obtener al cambiar la posición inicial colocando una capa de titanio sobre el sustrato (fig. 10b. Este proceso se suele llamar fotolitografía directa.

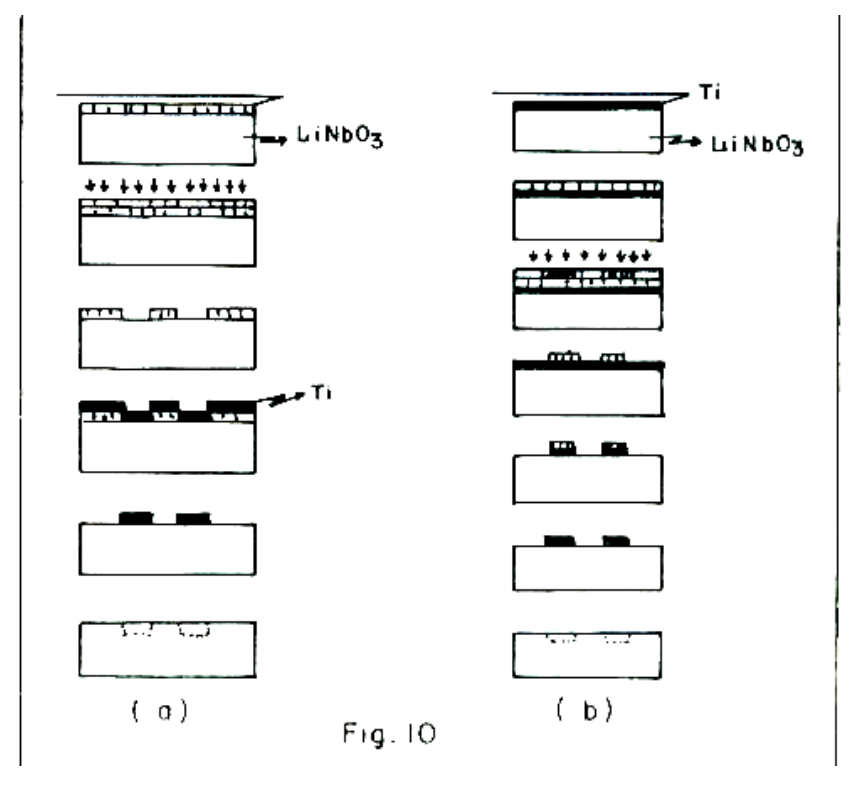

Fig. 10 
El principio de obtención de guías de banda con intercambio de iones es el mismo que para el caso anterior. Antes de sumergir los sustratos de vidrio se cubren con una capa de aluminio que obra como una barrera segura contra los iones durante el intercambio. Las capas de aluminio adhieren bien en el fundido caliente durante períodos suficientemente largos. Sobre esta capa se coloca un fotoresistor apropiado y luego el proceso se desarrolla según el esquema de la fig. 10a, hasta que en la capa metálica se abran los surcos requeridos. El intercambio iónico resulta solamente en las regiones no protegidas por el recubrimiento de aluminio. Después del intercambio el recubrimiento se quita químicamente. Al otro lado del sustrato se forma una guía plana para la cual las condiciones de intercambio iónico son idénticas a lo largo de ella. Hay que mencionar que el espesor de las bandas siempre resulta menor que el espesor de su correspondiente guía plana en el lado opuesto del sustrato. Mientras el índice de refracción máximo prácticamente no depende del ancho de la banda la profundidad disminuye monótonamente cuando la banda se estrecha.

La distribución transversal del índice de refracción en guías obtenidas por intercambio iónico depende de muchos factores:

- La composición del fundido (en régimen libre)

- El voltaje aplicado (cuando hay campo externo)

- Régimen temperatura-tiempo del tratamiento

- La composición del vidrio usado como sustrato

- Material de la máscara apantalladora, etc.

Si la máscara es conductora, que es el caso más frecuente, el ancho efectivo de la banda no difiere mucho de la abertura de la máscara ya que la difusión transversal se subordina a los campos eléctricos que aparecen. Sin embargo, en la práctica, en muchos casos la difusión libre da origen a un tamaño que puede sobrepasar varias veces el del canal de la máscara.

Junto a las dificultades de la fotolitografía y difusión que son inevitables, al elaborar guías de onda de $\mathrm{Ti}$ : $\mathrm{LiNbO} 3$ surgen otras dos. Una es la difusión inversa de $\mathrm{Li}_{2} 0$, ya mencionada, que conlleva a la formación de una guía plana que afecta las guías de banda que se desea obtener. Existen esperanzas de evitar este efecto realizando el proceso en una atmósfera de vapor de agua. La otra dificultad consiste en la destrucción de la guía cuando incide un haz de rayos la-ser de potencia mayor a $500 \mathrm{~W} / \mathrm{cm}^{2}$, que transforma los iones existentes de $\mathrm{Fe}^{2}$ en iones $\mathrm{Fe}^{3}$ liberando electrones cuya migración crea campos eléctricos internos a la vez que cambia localmente el índice de refracción. Este fenómeno se llama daño óptico o efecto foto-reactivo que se puede evitar utilizando radiación de longitud de onda más larga (como infarroja de 10,6 $\mu_{\mathrm{m}}$, donde la potencia alcanza más de $5 \mathrm{~kW} / \mathrm{cm}^{2}$

El uso de guías con materiales electroópticos sirve para crear condiciones de control y manejo de la luz. Con este fin se crea sobre la superficie del sustrato una configuración de electrodos apropiada. Esto requiere una fotomáscara adicional y su correspondiente ciclo de procedimientos fotolitográficos. Los electrodos son generalmente de aluminio de aproximadamente $200 \mathrm{~nm}$ de espesor lo que hace indispensable un acoplamiento muy preciso entre la máscara de los electrodos y las bandas ya formadas. A continuación se da una tabla con normas de inexactitudes permitidas, cuyo incumplimiento conlleva pérdidas de luz muy elevadas. 


\begin{tabular}{|l|l|}
\hline Espesor inicial de la capa de Ti & $<5 \mathrm{~nm}$ \\
Homogeneidad del espesor de la capa de Ti & $<1,5 \mathrm{~nm}$ \\
Constancia de la temperatura & $<2 \mathrm{~K}$ \\
Ancho de la guía & $<0,2 \mu \mathrm{m}$ \\
Suavidad de las arista de la guía & $<0,1 \mu \mathrm{m}$ \\
Radio de curvatura & $>30 \mathrm{~mm}$ \\
Angulo entre guías (de banda) y electrodos & $<0,01^{\circ}$ \\
Desviación paralela de los electrodos & $<0,5 \mu \mathrm{m}$ \\
\hline
\end{tabular}

\section{REFERENCIAS BIBLIOGRAFICAS}

1. Casas Justiniano, Optica, Cooperativa de Artes Gráficas, Zaragoza, 1983.

2. Carlson B., A foundry approach te intogratod optics, Photonicvs Spectra, pp. 152-1 53, Marzo1987.

3. Holman R.L., L.M. Althouse, DP. Skinner, Desirability of eloctrooptic materials for guided-wave optics. Optical Engineering. Vol. 26 No.2 pp 134-142. Feb. 1987. 